\title{
Group III - Nitride Semiconductors: Preeminent Materials for Modern Electronic and Optoelectronic Applications
}

\author{
Ananta R. Acharya \\ Department of Physics, Georgia Southern University, Statesboro, GA 30460 \\ Correspondence: anantaach@gmail.com
}

\begin{abstract}
Over the past two decades, group III-nitride semiconductors have become the focus of extremely intensive research due to their unique physical properties and their high potential for use in numerous electronic and optoelectronic devices. To date, almost all aspects of these materials have been explored, from understanding the fundamental physical properties to the development of fabrication technology of highly efficient devices for commercial use. In this article, some of the important physical properties and applications of III-nitride semiconducting materials have been presented.
\end{abstract}

Keywords: Wurtzit Structure, Nucleation Terahertz radiation devices, airbone application, low dark currents.

\section{INTRODUCTION}

A III-nitride compound semiconductor is formed due to the bonding of one of the group III elements such as, boron, aluminum, gallium or indium with the group V element, nitrogen. The III-nitrides and their alloys are direct band gap semiconductors. Their band gap varies from $0.7 \mathrm{eV}$ for indium nitride $(\mathrm{InN}), 3.4 \mathrm{eV}$ for gallium nitride $(\mathrm{GaN})$, to $6.2 \mathrm{eV}$ for aluminum nitride (AIN). Ternary III-nitride alloys like indium gallium nitride $\left(\operatorname{In}_{x} \mathrm{Ga}_{1-x} \mathrm{~N}\right)$, indium aluminum nitride (InAlN), and aluminum gallium nitride $(\mathrm{AlGaN})$ offer the tunable band gap which covers the whole visible spectrum and into the deep ultraviolet (UV) region. So, III-nitride alloys are particularly suitable for applications in optoelectronic devices for solid state lighting, such as laser diodes (LDs) and light emitting diodes (LEDs). High electron mobility and saturation velocity, high breakdown field, and high thermal conductivity are advantages of III-nitrides especially for highpower, high-speed electronics. The developments in the field of III-nitride semiconductors have been spectacular due to their highly attractive inherent properties. The outstanding achievements in the development of blue/ultraviolet (UV) LEDs, LDs and high-frequency transistors operating at high powers and temperatures [1-3] have proved the remarkable advantages of III-nitride semiconductor systems. The wide band gap energy range also makes these materials good candidates for absorber layers in solar cells since the absorption edge of these materials can be varied to optimize cell efficiency. III- nitride based electronic devices are also more environmentally friendly because they do not contain toxic elements such as arsenic that are used to fabricate other compound semiconductors such as gallium arsenide (GaAs).

In the recent years, the III-nitrides based research has attracted remarkable attention and gained a significant position in the science and technology of compound semiconductors due to a great number of possible applications of III-nitride semiconductors. $\mathrm{GaN}$ and its alloy $\operatorname{In}_{x} \mathrm{Ga}_{1-\mathrm{x}} \mathrm{N}$ have become dominant materials for producing high brightness LEDs and LDs that emit light in the green/blue region of the visible spectrum. $\operatorname{In}_{x} \mathrm{Ga}_{1-\mathrm{x}} \mathrm{N}$ based light emitting diodes are already in use in full color liquid crystal diode (LCD) displays and traffic lights. All these applications demonstrate the technological relevance of the III-nitride compounds and the reason for these materials to be the subject of an active research field.

\section{PROPERTIES}

The binary group III-nitrides AlN, GaN and InN can crystallize in three structural phases; (a) wurtzite, (b) zincblende and (c) rock-salt $(\mathrm{NaCl})$. However, wurtzite structure is the thermodynamically stable phase at ambient conditions [4-6]. The zincblende structure is metastable and can be stabilized by heteroepitaxial thin film growth on the (001) crystal planes of cubic structures such as $\mathrm{Si}, \mathrm{MgO}$ and $\mathrm{GaAs}$ 
[7]. A phase transition to the rock-salt structure takes place at high pressure [7]. The wurtzite and zincblende structures are similar in many aspects. In both cases, the group III element is surrounded by four atoms of the group $\mathrm{V}$ element, which are arranged at the edges of a tetrahedron. Likewise, the group $\mathrm{V}$ element is surrounded by four atoms of group III elements. Also, the twelve nextnearest neighbors are at the equal distance in both structures. The main difference between wurtzite and zincblende structure is found only from the third next nearest neighbors, that is, they have different stacking sequences. The stacking sequence of the wurtzite structure along [0001] is ABAB, while that of zincblende structure along [001] direction is $\mathrm{ABCABC}$, where $\mathrm{A}, \mathrm{B}$, and $\mathrm{C}$ refer to allowed sites of the III-N pairs of the closed-packed layers. A fault in stacking sequence may transform one structure into another or create a structural defect. The wurtzite structure can be transformed into the rock-salt structure at high pressures. In this transformation, the bond character changes mostly from covalent to ionic and from four to six fold coordination of atoms. The structural phase transition was experimentally observed at pressures of $22.9 \mathrm{GPa}, 52.2 \mathrm{GPa}$ and $12.1 \mathrm{GPa}$ for AlN, GaN and $\mathrm{InN}$, respectively [8-10].

As mentioned above, the III-nitride semiconductors and their alloys are direct band gap semiconductors. The band gap energy of a semiconductor is an important parameter that determines its transport and optical properties as well as many other phenomena. The major technological advantage of the III-nitride system is that by alloying $\mathrm{GaN}$ with $\mathrm{InN}$ and/or AlN the band gap can be tuned in a controllable fashion covering a wide spectral range from deep UV (AIN $=6.2 \mathrm{eV})$ to near IR $(\mathrm{InN}=0.7 \mathrm{eV})$. So, ternary and quaternary alloy systems of AlN, GaN and InN (such as InGaN, InAlN, AlGaN, and AlGAInN) can have continuous band gap energy from $0.7 \mathrm{eV}$ to 6.2 $\mathrm{eV}$ depending upon the composition of In or $\mathrm{Ga}$ or $\mathrm{Al}$ in the alloy system.

A unique characteristic inherent in III-nitrides is the strong polarization $[11,12]$. Due to the lack of a center of symmetry and the large ionicity of the metal-nitrogen bonds, spontaneous polarization is present along the c-axis in the wurtzite structure of the III-nitride materials. Also, the introduction of an external strain on this structure produces piezoelectric polarization. So, the net polarization is composed of two parts: (1) spontaneous, which is inherent to the material and (2) strain-induced piezoelectricity; both are influenced by the polarity of the III-nitrides. Spontaneous and piezoelectric polarizations present in wurtzite III-nitrides influence the optical and electrical properties of the material.

The chemical bonding within III-nitrides is very strong. Due to this strong chemical bonding, III-nitride semiconductors have a high melting point, mechanical strength and chemical stability. In addition, their strong bonding makes them resistant to high-current electrical degradation and radiation damage that is present in the active regions of light emitting devices [13]. These materials also possess good thermal conductivity. IIInitride based devices can operate at high temperatures as well as in hostile environments.

The performance and efficiency of III-nitrides based electronic devices depend upon the electronic properties, particularly carrier concentration and mobility. A range of values of electron mobility and background concentration have been measured for AlN, GaN, and InN by using Hall measurements. Carrier mobility depends upon various factors such as temperature, electric field, doping concentration and material quality of the semiconductors. If the lattice mismatch is large then the value of carrier mobility is low. Using a buffer layer during the nucleation reduces the strain which enhances the carrier mobility.

The optical properties of the III-nitride based devices depend upon some important factors such as refractive index and dielectric constant of the materials. Some important structural, electronic, thermal and optical properties of wurtzite AlN, GaN and $\mathrm{InN}$ are listed in Table 1. 
Table 1 Properties of wurtzite III-nitride materials., 14-21

\begin{tabular}{|c|c|c|c|}
\hline Property & AlN & $\mathrm{GaN}$ & $\mathrm{InN}$ \\
\hline \multicolumn{4}{|l|}{$\begin{array}{l}\text { Thermal expansion coefficient } \\
\qquad(300 \mathrm{~K})\end{array}$} \\
\hline $\mathrm{a}\left(\times 10^{-6} \mathrm{~K}^{-1}\right)$ & 4.2 & 5.59 & 5.7 \\
\hline $\mathrm{c}\left(\times 10^{-6} \mathrm{~K}^{-1}\right)$ & 5.3 & 3.17 & 3.7 \\
\hline Thermal conductivity $\left(\mathrm{Wcm}^{-1} \mathrm{~K}^{-1}\right)$ & 2.0 & 1.3 & 0.8 \\
\hline Melting point $\left({ }^{\circ} \mathrm{C}\right)$ & $>3000$ & $>2500$ & $>1100$ \\
\hline Bulk modulus (GPa) (300K) & 210 & $210 \pm 10$ & 140 \\
\hline Refractive index & 2.2 & 2.35 & 2.56 \\
\hline Bond length $(\AA)$ & 1.89 & 1.94 & 2.15 \\
\hline Cohesive energy per bond $(\mathrm{eV})$ & 2.88 & 2.24 & 1.93 \\
\hline Lattice constant $(\AA)$ & a3.11, ${ }^{\circ} 4.97$ & a3.18, c5.18 & ${ }^{\mathrm{a}} 3.54,{ }^{\mathrm{c}} 5.70$ \\
\hline Energy gap $(\mathrm{eV})$ & 6.2 & 3.39 & 0.7 \\
\hline Effective electron mass $\left(\mathrm{m}_{\mathrm{o}}\right)$ & 0.48 & 0.2 & 0.06 \\
\hline \multirow[t]{2}{*}{ Electron mobility $\left(\mathrm{cm}^{2} \mathrm{~V}^{-1} \mathrm{~s}^{-1}\right)$} & 300(Theo.) & 1000 (Theo.) & 14000(Theo.) \\
\hline & 426 (Expt.) & 900(Expt.) & 3980(Expt.) \\
\hline Peak drift velocity $\left(\times 10^{7} \mathrm{~cm} \mathrm{~s}^{-1}\right)$ & 0.02 & 3.1 & 4.3 \\
\hline Electron concentration $\left(\mathrm{cm}^{-3}\right)$ & $<10^{16}$ & $\sim 10^{17}$ & $>10^{19}$ \\
\hline \multirow[t]{2}{*}{ Dielectric constant } & $\varepsilon_{0}=8.5 \pm 0.2$ & $\varepsilon_{0}=10.0$ & $\varepsilon_{0}=15.3$ \\
\hline & $\varepsilon_{\infty}=4.68-4.84$ & $\varepsilon_{\infty}=5.5$ & $\varepsilon_{\infty}=8.4$ \\
\hline
\end{tabular}

\section{APPLICATIONS}

In recent years, III-nitride semiconductors have gained a significant position in science and technology due to their potential for numerous device applications. The majority of such devices can be divided into two categories: electronic and optoelectronic devices. III-nitride semiconductors, particularly AlN and GaN have very high melting point (see the table 1). Due to this property, they can be used in high power and high temperature electronic devices such as, automotive, aerospace and power industries. High temperature electronics allow control directly in harsh environments, such as in engines, making them important in the automotive and aerospace industries. Since IIInitride semiconductors can operate adequately bearing high temperature at the same time, these electronics can be operated at high temperature without using any cooling system. This helps minimize the cost as well as the weight and size of the systems.
III-nitride semiconductors based UV photodetectors are sensitive to UV radiation while being insensitive to longer wavelength radiation. Such devices have applications where there is a need to detect or control the source of UV radiation in an existing background of visible or infrared radiation [22]. For example, such devices can be used for flame detection, furnace and engine monitoring for automotive, aerospace and petroleum industry, undersea communications, space-to-space communications secure from the Earth, early missile threat warning and portable battlefield reagent/chemical analysis system. IIInitride based devices are expected to work without optical filters and complex electronics due to their intrinsic solar blindness and low dark currents. This reduces the launch weight significantly for space and airborne applications. III-nitride based blue and green light emitting diodes (LEDs) have already been used commercially. When used with the red AlGaAs based LEDs, the LEDs complete the primary colors (red, green, and blue) for large, high brightness, outdoor full-color displays. Green 
LEDs are used in traffic lights because of their superior efficiency and reliability as compared to incandescent light sources. Solid state white light sources using a combination of red, green and blue LEDs may soon replace the conventional light bulbs with better efficiency and reliability. Currently, high performance photovoltaics are exclusively based on Ge/GaAs/GaInP multijunctions. Conversion efficiencies of over $30 \%$ have been reported on $\mathrm{GaInP} / \mathrm{GaAs} / \mathrm{Ge}$ triple junction devices [23]. $\operatorname{In}_{x} \mathrm{Ga}_{1-x} \mathrm{~N}$, a ternary alloy of InN and GaN, has many material properties that make it an excellent candidate for high efficiency PV devices. In $\mathrm{Ga}_{1-\mathrm{x}} \mathrm{N}$ alloys have been shown to have superior high energy radiation resistance for space based PV applications [24]. Recent studies on InN reveal that the band gap of the $\mathrm{In}_{\mathrm{x}} \mathrm{Ga}_{1-\mathrm{x}} \mathrm{N}$ material system spans continuously ranging from $0.6 \mathrm{eV}$ to $3.4 \mathrm{eV}[25,26]$, which has an almost perfect match to the terrestrial solar spectrum, thus enabling design of multijunction solar cell structures with near ideal band gaps for maximum efficiency. Even more interesting is that the band gap is direct for the entire material system. The III-nitride alloys also tend to exhibit very strong absorption of approximately $105 \mathrm{~cm}^{-1}$ at the band edge [27], allowing a large fraction of the incident light to be absorbed in a hundred nanometers of material. This is in contrast to the tens or hundreds of microns of material as is necessary in traditional Si solar cells [28].

Due to the superior properties over other semiconducting materials, the III-nitrides have also shown utility in the development of UV and IR detectors [29], room temperature spintronic applications [30], high electron mobility transistors [31], chemical and biological sensors [32], thermoelectric devices [33], terahertz radiation devices [34], and more recently nuclear detection [35].

\section{SUMMARY}

In this article, some of the important properties and applications of III-nitride semiconductors have been discussed. The III-nitride semiconductors have unique physical properties such as, high electron mobility, high saturation velocity, direct and tunable band gap, high absorption coefficient, and high thermal stability and conductivity. Due to these outstanding physical properties, III-nitride semiconductors are the promising materials for numerous electronic and optoelectronic device applications such as, LEDs, LDs, solar cells, high electron mobility transistors, sensors, terahertz devices, and high-temperature thermoelectric devices. However, there are still many scientific and technological challenges that need to be addressed. For instance it is difficult to control defects owing to lack of native substrates. Incorporating a high concentration of indium into the $\operatorname{In}_{x} \mathrm{Ga}_{1-\mathrm{x}} \mathrm{N}$ alloys remains challenging due to phase separation and the lattice mismatch between $\mathrm{GaN}$ and $\mathrm{InN}$. Nevertheless, despite the challenges mentioned above, the scientific importance of continuing to develop III-nitride-based devices is evident, and the outlook is promising.

\section{REFERENCES}

[1] S. Strite and H. Morkoc, J. Vac. Sci. Technol. B 10, 1237 (1992).

[2] S. N. Mohammad and H. Morkoc, Prog. Quantum Electron. 20, 361 (1996).

[3] O. Ambacher, J. Phys. D: Appl. Phys. 31, 2653 (1998).

[4] I. Akasaki, H. Amano, in Group III nitrides, ed J. H. Edgar, INSPEC, UK, pp. 30-34 (1994).

[5] W. J. Meng, in Group III nitrides, ed J. H. Edgar, INSPEC, UK pp.22-29 (1994).

[6] T. L. Tansley, in Group III nitrides, ed J. H. Edgar, INSPEC, UK pp.35-42 (1994).

[7] B. Gil, Group III nitride semiconductor compounds Physics and applications; Clarendon Press, Oxford (1998).

[8] P. Perlin, C. Jauberthiecarillon, J. P. Itie, A. S. Miguel, I. Grzegory, A. Polian, Phys. Rev.B 45, 83 (1992).

[9] M. Ueno, M. Yoshida, A. Onodera, O. Shimomura, K. Takemura, Phys. Rev. B 49, 14 (1994). 
[10] Q. Xia, H. Xia, A. L. Ruoff, J. Appl. Phys. 73, 8198 (1993).

[11] F. Bernardini and V. Fiorentini, Phys. Stat. Sol.b 216, 391 (1999).

[12] F. Bernardini and V. Fiorentini, Phys. Rev. b 64, 085207 (2001).

[13] F. A. Ponce, and D. P. Bour, Nature 386, 351 (1997).

[14] F. A. Ponce, Introduction to Nitride Semiconductor Blue Lasers and Light Emitting Diodes, Ed. by S. Nakamura and S. Chichibu (Taylor and Francis, Ltd., London, 2000), Chapter 4.

[15] I. Vurgaftman and J. R. Meyer, J. Appl. Phys. 94, 3675 (2003).

[16] V. M. Polyakov and F. Schweirz, Appl. Phys. Lett. 88, 032101 (2006).

[17] S. N. Mohammad, H. Morkoc, Prog. Quant. Electr. 20, 361 (1996).

[18] K. Matsubara, T. Takagi, Jpn. J. Appl. Phys. 22, 511 (1982).

[19] A. S. Barker, M. Illegems, Phys. Rev. B 7, 743 (1973).

[20] F. Bechstedt, B. Gil (Ed.), Low Dimensional Nitride Semiconductors, Oxford University Press, P.11 (2002).

[21] F. Bernadini, V. Fiorentini, D. Vanderbilt, Phys. Rev. Lett. 79, 3958 (1997).

[22] M. Razeghi and A. Rogalski, J. Appl. Phys. 79, 7433 (1996).

[23] T. Takamoto, E. Ikeda, H. Kurita, and M. Ohmori, Appl. Phys. Lett. 70, 381 (1997).
[24] J. Wu, W. Walukiewicz, K. M. Yu, W. Shan, J. W. Ager III, E. E. Haller, H. Lu, W. J. Schaff, W. K. Metzger, and S. Kurtz, J. Appl. Phys. 94, 6477 (2003).

[25] J. Wu, W. Walukiewicz, K. M. Yu, J. W. Ager, E. E. Haller, L. Hai, and W. J. Schaff, Appl. Phys. Lett. 80, 4741 (2002).

[26] T. Matsuoka, H. Okamoto, M. Nakao, H. Harima, and E. Kurimoto, Appl. Phys. Lett. 81, 1246 (2002).

[27] J. F. Muth, J. H. Lee, I. K. Shmagin, R. M. Kolbas, J. Casey, B. P. Keller, U. K. Mishra, and S. P. DenBaars, Appl. Phys. Lett. 71, 2572 (1997).

[28] D. Redfield, Appl. Phys. Lett. 25, 647 (1974).

[29] Ariyawansa G et. al. Phys. Lett. 89, 0191113 (2006).

[30] Liu Z, Yi X, Wang J, Kang J, Melton A G, Shi Y, Lu N, Wang J and Li, J. Appl. Phys. Lett. 100, 23408 (2012).

[31] M. Singh, J. Singh, J. Appl. Phys. 94, 2498 (2003).

[32] H. Lu, W.J. Schaff, L.F. Eastman, J. Appl. Phys. 96, 3577 (2004).

[33] S. Yamaguchi, R. Izaki, N. Kaiwa, S. Sugimura, A. Yamamoto, Appl. Phys. Lett. 84, 5344 (2004).

[34] Y.M. Meziani, B. Maleyre, M.L. Sadowski, S. Ruffenach, O. Briot, W. Knap, Phys. Status Solidi (a) 202, 590 (2005).

[35] Melton A G, Burgett E, Xu T, Hertel N and Ferguson I T, Phys. Status Solidi (c) 9, 957 (2012). 